\section{Battle's Sign}

Beth Rush

Psychiatry and Psychology, Mayo Clinic, Jacksonville, FL, USA

\section{Synonyms}

Periauricular or mastoid ecchymosis

\section{Definition}

Named after English surgeon, Dr. William Henry Battle, this is a clinical symptom suggestive of basilar skull/middle cranial fossa fracture. After blunt force head trauma, leaking of blood from the blood vessels in the skull, typically the posterior auricular artery, leads to a crescent-shaped bruise wrapping behind the base of the earlobe and extending posteriorly toward the point of the neck where the base of the skull meets the neck. A patient with this symptom may present with acute bloody discharge of the ear and/or nose. Battle's sign may occur a few days following the onset of the skull fracture.

\section{Cross-Reference}

- Depressed Skull Fracture

\section{References and Readings}

Victor, M., \& Ropper, A. H. (2001). Principles of neurology (7th ed.pp. 925-953). New York: McGraw-Hill. 